care-associated infections and deaths in U.S. hospitals, 2002. Public Health Rep 2007;122(2):160-166.

8. Harbarth S, Sax H, Gastmeier P. The preventable proportion of nosocomial infections: an overview of published reports. $J$ Hosp Infect 2003;54(4):258-266.

9. Scott RD. The direct medical costs of healthcare-associated infections in US hospitals and the benefits of prevention. http:// www.cdc.gov/ncidod/dhqp/pdf/Scott_CostPaper.pdf. Accessed June 2, 2011.

10. Graves N, Harbarth S, Beyersmann J, Barnett A, Halton K, Cooper B. Estimating the cost of health care-associated infections: mind your p's and q's. Clin Infect Dis 2010;50(7): 1017-1021.

\section{Low Adherence to Outpatient Preoperative Methicillin-Resistant Staphylococcus aureus Decolonization Therapy}

To the Editor-Evidence supports methicillin-resistant Staphylococcus aureus (MRSA) decolonization with topical antimicrobial and antiseptic agents to prevent infections in select patient groups. ${ }^{1-5}$ While therapy effectiveness is heavily impacted by adherence, there is a lack of data on patientreported adherence to such therapy., ${ }^{4,6,7}$ Here we report outpatient adherence to preoperative MRSA decolonization therapy obtained from nursing-administered surveys.

In 2006, the Providence Veterans Affairs Medical Center implemented a preoperative MRSA colonization surveillance and decolonization program. MRSA nares screening was conducted during surgical scheduling appointments from January 2006 to December 2009. Patients were provided 15 minutes of nursing education on MRSA, what to anticipate if MRSA-positive, appropriate decolonization therapy application techniques, and date-specific time lines featuring dayby-day instructions for use prior to surgery.

Surgical Service nurse practitioners contacted MRSAcolonized outpatients a minimum of 7 days prior to surgery to notify them of their screening results, to remind them they would be receiving the decolonization package in the mail or remind them to pick it up from the pharmacy, and to review application techniques and time lines for use. The decolonization regimen was prescribed as follows: mupirocin $2 \%$ ointment to both nares twice daily for 5 days prior to surgery and use of hexachlorophene $3 \%$ or chlorhexidine gluconate $4 \%$ body wash once daily for 3 days prior to surgery.

On the day of surgery, patients were rescreened and administered adherence surveys by nursing to ascertain the number of days each therapy was applied. Proportion of days covered (PDC) was calculated as the number of days therapy was applied, divided by the number of prescribed days of therapy. Complete adherence to the decolonization regimen was defined as a PDC of 1.0 for both mupirocin (5/5 days) and body wash (3/3 days). Colonization persistence was defined as a positive nares culture on the day of surgery. Postoperative MRSA infections were identified from positive clinical cultures in addition to a physician diagnosis of infection and/or nursing notes describing clinical signs of infection in the 30 days following surgery. We assessed differences in colonization persistence and postoperative MRSA infections at different PDC levels with $\chi^{2}$ and Fisher exact tests as appropriate.

Mupirocin susceptibilities were available for a sample of the MRSA-positive preoperative nares screening isolates, and resistance was defined as low level (minimum inhibitory concentration, $8-128 \mathrm{mg} / \mathrm{L}$ ) or high level $(\geq 256 \mathrm{mg} / \mathrm{L})$ according to previously described methods. ${ }^{8}$ We assessed PDC temporal trends by using nonparametric Spearman rank correlation. All analyses were performed using SAS, version 9.1.3 (SAS Institute). This study was reviewed and approved by the Institutional Review Board.

Of the 45 MRSA-colonized outpatients who received the preoperative decolonization kit, $62.2 \%$ applied mupirocin to their nares as instructed for 5 days prior to their scheduled surgery (Table 1). Body wash was applied for 3 days by $46.7 \%$ of patients. Most patients were male ( 1 female), with a mean age of 57 years (standard deviation, 19). Surgery types included various noncardiothoracic surgeries, the majority of which were orthopedic, vascular, urological, hernia repairs, or tumor resections.

Complete adherence to the decolonization regimen was reported by $31.1 \%$ of patients (Table 1 ). The most common patient-reported reason for incomplete adherence related to recall, as several patients could not remember whether and when they applied each topical therapy. Five (11.1\%) patients developed a postoperative MRSA infection in the 30 days following surgery, and $17(37.8 \%)$ patients were still colonized on the day of surgery. Colonization persistence and 30-day MRSA infections did not vary significantly by PDC $(P \geq$ .10 for all comparisons; Table 1). Body wash PDC decreased significantly over the study period $(P<.03)$. No temporal trends were observed in mupirocin PDC, adherence to both mupirocin and body wash, colonization persistence, or MRSA infections.

Mupirocin susceptibilities of preoperative nares screening isolates were available for $40.0 \%$ (18/45) of patients. Five $(27.8 \%)$ of the available isolates were mupirocin resistant (4 high level, 1 low level). Four of 5 patients with mupirocinresistant isolates were still colonized on the day of surgery ( 3 high level, 1 low level). Only 1 patient with mupirocinresistant MRSA (high level) developed a MRSA infection in the 30 days following surgery. This patient was $100 \%$ adherent to mupirocin, with $0 \%$ adherence to body wash. When patients colonized with mupirocin-resistant MRSA were excluded, no differences were observed in colonization persistence or follow-up MRSA infections by PDC.

Nearly two-thirds of patients completed mupirocin therapy as instructed, while only one-third were fully adherent to both 
TABLE 1. Methicillin-Resistant Staphylococcus aureus (MRSA) Decolonization Therapy: Proportion of Days Covered (PDC), Colonization Persistence, and Postoperative MRSA Infections

\begin{tabular}{lccc}
\hline & Mupirocin & Body wash & Mupirocin and body wash \\
\hline Mean PDC (SD) & $0.8(0.3)$ & $0.7(0.4)$ & $\ldots$ \\
Median PDC (IQR) & $1.0(0.6-1.0)$ & $0.7(0.3-1.0)$ & $\ldots$ \\
PDC $=1.0^{\text {a }}$ & $28(62.2)$ & $21(46.7)$ & $14(31.1)$ \\
$\quad$ Colonized on day of surgery & $8(28.6)$ & $9(42.9)$ & $5(35.7)$ \\
30-day postoperative MRSA infection & $3(10.7)$ & $2(9.5)$ & $1(7.1)$ \\
PDC $\geq 0.8^{b}$ & $30(66.7)$ & $21(46.7)$ & $16(35.6)$ \\
Colonized on day of surgery & $9(30.0)$ & $9(42.9)$ & $6(37.5)$ \\
30-day postoperative MRSA infection & $4(13.3)$ & $2(9.5)$ & $2(12.5)$ \\
PDC $\geq 0.7^{c}$ & $30(66.7)$ & $28(62.2)$ & $19(42.2)$ \\
Colonized on day of surgery & $9(30.0)$ & $10(35.7)$ & $6(31.6)$ \\
30-day postoperative MRSA infection & $4(13.3)$ & $3(10.7)$ & $3(15.8)$ \\
PDC $\geq 0.6^{d}$ & $37(82.2)$ & $28(62.2)$ & $8(55.6)$ \\
Colonized on day of surgery & $12(32.4)$ & $10(35.7)$ & $3(12.0)$ \\
30-day postoperative MRSA infection & $4(10.8)$ & $3(10.7)$ & \\
\hline
\end{tabular}

Nоте. Data are no. (\%) of patients, unless otherwise indicated. IQR, interquartile range; SD, standard deviation.

${ }^{a}$ Mupirocin 5/5 days and body wash $3 / 3$ days.

b Minimum of mupirocin 4/5 days and body wash $3 / 3$ days.

c Minimum of mupirocin 4/5 days and body wash 2/3 days.

Minimum of mupirocin 3/5 days and body wash $2 / 3$ days.

mupirocin and body wash. In the hospital setting, previously reported institutional adherence estimates range from moderately high $(75 \%)$ to relatively low $(37 \%)$, similar to our patient-reported adherence in the outpatient setting.,7 This is the first observational study describing patient-reported adherence to outpatient preoperative MRSA decolonization therapy based on our review of the literature.

Colonization persistence and postoperative MRSA infections did not vary by adherence, although our study population was small, with few follow-up MRSA infections, impacting our ability to detect significant differences between adherent and nonadherent patients. Our findings are also limited by the incomplete data on mupirocin susceptibilities since colonization persistence was observed in $80 \%$ of patients with mupirocin-resistant MRSA. Adherence may have been underestimated due to recall. Providing patients a formal dayby-day decolonization therapy checklist to be returned on the day of surgery may improve recall and adherence.

In conclusion, despite comprehensive education, patientreported adherence to preoperative MRSA decolonization therapy was low $(31.1 \%)$ in the outpatient setting. While MRSA infections and colonization persistence were similar between adherent and nonadherent outpatients, adherence may affect therapy effectiveness and should be considered by those implementing similar preoperative MRSA decolonization programs.

\section{ACKNOWLEDGMENTS}

We gratefully acknowledge the nursing staff of the Ambulatory Diagnostics and Treatment Unit (ADTU/4B) at the Providence Veterans Affairs Medical Center for education and survey administration.
Financial support. A.R.C. is supported by a Department of Veterans Affairs Career Development Award.

Potential conflicts of interest. All authors report no conflicts of interest relevant to this article.

Aisling R. Caffrey, MS, $\mathrm{PhD}^{1,2}$

Suzanne B. Woodmansee, BS, RN; ${ }^{1}$ Nancy Crandall, NP; ${ }^{3}$ Cynthia Tibert, BSN, $\mathrm{CIC}_{;}^{4}$ Corinne Fielding, $\mathrm{RN} ;{ }^{5}$

Dennis J. Mikolich, MD; ${ }^{6,7}$ Michael P. Vezeridis, MD; $;^{3,8}$ Kerry L. LaPlante, PharmD ${ }^{1,2,6}$

Affiliations: 1, Infectious Diseases Research Program, Providence Veterans Affairs Medical Center, Providence, Rhode Island; 2. Department of Pharmacy Practice, University of Rhode Island, Kingston, Rhode Island; 3. Surgical Service, Providence Veterans Affairs Medical Center, Providence, Rhode Island; 4. Infection Control, Veterans Affairs Boston Healthcare System, West Roxbury, Massachusetts; 5. Infection Control, Providence Veterans Affairs Medical Center, Providence, Rhode Island; 6. Department of Infectious Diseases, Alpert Medical School of Brown University, Providence, Rhode Island; 7. Infectious Diseases, Providence Veterans Affairs Medical Center, Providence, Rhode Island; 8. Department of Surgery, Alpert Medical School of Brown University, Providence, Rhode Island.

Address correspondence to Kerry L. LaPlante, PharmD, Veterans Affairs Medical Center (151), Research Building 35, 830 Chalkstone Avenue, Providence, RI 02908 (kerrytedesco@uri.edu).

Presented in part: 50th Annual Interscience Conference on Antimicrobial Agents and Chemotherapy; Boston, MA; September 14, 2010.

The views expressed are those of the authors and do not necessarily reflect the position or policy of the US Department of Veterans Affairs. Infect Control Hosp Epidemiol 2011;32(9):930-932

(C) 2011 by The Society for Healthcare Epidemiology of America. All rights reserved. 0899-823X/2011/3209-0019\$15.00. DOI: $10.1086 / 661787$

\section{REFERENCES}

1. Ammerlaan HS, Kluytmans JA, Wertheim HF, Nouwen JL, Bon- 
ten MJ. Eradication of methicillin-resistant Staphylococcus aureus carriage: a systematic review. Clin Infect Dis 2009;48:922-930.

2. van Rijen MM, Bonten M, Wenzel RP, Kluytmans JA. Intranasal mupirocin for reduction of Staphylococcus aureus infections in surgical patients with nasal carriage: a systematic review. J Antimicrob Chemother 2008;61:254-261.

3. Kallen AJ, Wilson CT, Larson RJ. Perioperative intranasal mupirocin for the prevention of surgical-site infections: systematic review of the literature and meta-analysis. Infect Control Hosp Epidemiol 2005;26:916-922.

4. Robicsek A, Beaumont JL, Thomson RB Jr, Govindarajan G, Peterson LR. Topical therapy for methicillin-resistant Staphylococcus aureus colonization: impact on infection risk. Infect Control Hosp Epidemiol 2009;30:623-632.

5. Infectious Diseases Society of America, Emerging Infections Network. Perioperative Staphylococcus aureus Screening and Decolonization. Arlington, VA: Infectious Diseases Society of America, 2010.

6. Strom B, Kimmel S, eds. Textbook of Pharmacoepidemiology. Chichester: Wiley, 2006.

7. Hansen D, Patzke PI, Werfel U, Benner D, Brauksiepe A, Popp W. Success of MRSA eradication in hospital routine: depends on compliance. Infection 2007;35:260-264.

8. Caffrey AR, Quilliam BJ, LaPlante KL. Risk factors associated with mupirocin resistance in methicillin-resistant Staphylococcus aureus. J Hosp Infect 2010;76:206-210.

\section{Impact of PCR Testing for Clostridium difficile on Incident Rates and Potential on Public Reporting: Is the Playing Field Level?}

To the Editor-Healthcare-associated infections are a challenge to the healthcare field that pose an impact on the outcomes of patients. Preventive measures are implemented at most healthcare institutions to minimize the risks of acquiring healthcare-associated infections. ${ }^{1}$ Clostridium difficile is a fastidious anaerobic organism that is primarily responsible for antibiotic-associated colitis and accounts for about one-quarter of nosocomial antibiotic-associated diarrhea. ${ }^{2}$ In response to a recognized increase in disease activity and severity, the Ohio Department of Health made C. difficile infection (CDI) a reportable disease in 2006, ${ }^{3}$ and it was reinstated under the revised House Bill 197 in August 2010. While the benefits to transparency are numerous, the downside is that rates will be used for interhospital comparison, despite lack of adjustment for case mix. ${ }^{4}$ The intersection of public reporting for $\mathrm{CDI}$ in Ohio and the advent of testing based on polymerase chain reaction (PCR) adds another layer of complexity. On October 19, 2010, the Cleveland Clinic changed testing from enzyme immunoassay to a PCR test for $C$. difficile detection. We sought to compare our CDI rates before and after the institution of PCR-based testing and evaluate the effect this will have on our mandate for public reporting.
TABLE 1. Results of Testing of Consecutive Stool Samples for Clostridium difficile Using Enzyme Immunoassay (EIA) Toxin and Polymerase Chain Reaction (PCR) during a 3Month Period

\begin{tabular}{lccc}
\hline & EIA & PCR & $P$ \\
\hline No. of lab specimens & 2,579 & 2,534 & \\
Mean no. (\%) positive & $167(6.5)$ & $382(15.1)$ & $<.001$ \\
CDI rates $^{\mathrm{a}}$ & 4.9 & 10.3 & $<.001$ \\
\hline
\end{tabular}

NOTE. EIA toxin used before October 19, 2010, and PCR used after. CDI, Clostridium difficile infection.

a Cases per 10,000 patient-days.

CDI surveillance is performed prospectively at the Cleveland Clinic by infection preventionists. Cases are ascertained by daily review of lab reports of patients with positive stool tests for $C$. difficile, and chart review establishes the presence and onset of symptoms. Before October 19, 2010, testing was performed by enzyme immunoassay detection of toxins $A$ and $\mathrm{B}$ (Wampole), and after this date, the testing methodology was changed to PCR (BD Genehom). Query of the microbiology information systems was done to compare the results of testing for $\mathrm{CDI}$, using consecutive stool samples during a 3-month period before and after the change. A telephone survey of 11 other Ohio hospitals was conducted to determine whether PCR testing for CDI had been implemented at their institution.

The prevalence of positive tests for CDI increased significantly from $6.5 \%$ of 2,579 stools tested to $15.1 \%$ of 2,534 stools tested after introduction of PCR testing $(P<.001)$. The rate of CDI also increased significantly (from 4.9 per 10,000 patient-days to 10.3 ; Table 1 ). There was no identified $C$. difficile cluster after implementation of PCR testing to account for the increased percent positives observed. None of the 11 hospitals in Ohio contacted had introduced PCR testing for CDI during this time. There was a significant increase in the number and rates of CDI after the introduction of PCR testing that was unexplained by other reasons and not unexpected. Decisions about choice of diagnostic platform for CDI testing are complex and should not be driven by need for publicly reporting rates. However, public display of CDI rates are an implicit comparison of quality of care provided. In the case of CDI, identifying test methods should be included.

\section{ACKNOWLEDGMENTS}

Potential conflicts of interest. All authors report no conflicts of interest relevant to this article.

Kimberlee S. Fong, DO; ${ }^{1}$ Cynthia Fatica, RN, BSN; ${ }^{2}$ Geraldine Hall, PhD; ${ }^{3}$ Gary Procop, $\mathrm{MD}^{3}$

Susan Schindler, M(ASCP); $;^{3}$ Steven M. Gordon, MD; ${ }^{1}$ Thomas G. Fraser, MD

Affiliations: 1. Cleveland Clinic Department of Infectious Disease, Cleve- 